\title{
Caveats in obtaining high-quality 2D materials and property characterization
}

\author{
Shruti Subramanian ${ }^{1, b)}$, Natalie Briggs ${ }^{2, b)}$ (D), Jeffrey Shallenberger ${ }^{3}$, Maxwell \\ T. Wetherington ${ }^{1,3}$, Joshua A. Robinson ${ }^{4, a)}$ \\ ${ }^{1}$ Department of Materials Science \& Engineering, The Pennsylvania State University, University Park, Pennsylvania 16802, USA \\ ${ }^{2}$ Department of Materials Science \& Engineering, The Pennsylvania State University, University Park, Pennsylvania 16802, USA; and 2-Dimensional \\ Crystal Consortium, The Pennsylvania State University, University Park, Pennsylvania 16802, USA \\ ${ }^{3}$ Materials Characterization Lab, The Pennsylvania State University, University Park, Pennsylvania 16802, USA \\ ${ }^{4}$ Department of Materials Science \& Engineering, The Pennsylvania State University, University Park, Pennsylvania 16802, USA; 2-Dimensional Crystal \\ Consortium, The Pennsylvania State University, University Park, Pennsylvania 16802, USA; Materials Research Institute, The Pennsylvania State \\ University, University Park, Pennsylvania 16802, USA; and Center for Atomically Thin Multifunctional Coatings, The Pennsylvania State University, \\ University Park, Pennsylvania 16802, USA \\ ${ }^{a)}$ Address all correspondence to this author. e-mail: jrobinson@psu.edu \\ b) These authors contributed equally to this work. \\ This paper has been selected as an Invited Feature Paper. \\ Received: 12 November 2019; accepted: 6 December 2019
}

The field of two-dimensional (2D) materials remains a key area of scientific research today, generating continual interest for electronic, sensing, and quantum technology. As the field progresses beyond proof-of-concept devices, experimental and analytical methods and results must be scrutinized to ensure the veracity of scientific claims. Here, some favored synthesis and characterization techniques within the 2D material (2DM) community and certain limitations inherent to these techniques are discussed. The authors highlight select caveats of solidsource and seed-promoted synthesis techniques, such as difficulties in reproducibility and compromised electrical performance of films synthesized with nucleation agents. Furthermore, the importance of careful characterization methodology in determining 2DM layer number, stoichiometry, and dopant effects is discussed. This article is intended to further educate researchers regarding select techniques and claims in the 2DMs field.

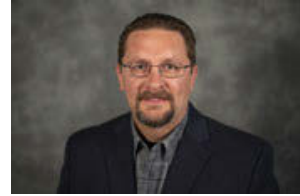

Joshua A. Robinson
Dr. Robinson obtained his B.S. degree in Physics with minors in Chemistry and Mathematics from Towson University in 2001. He received his doctorate degree from The Pennsylvania State University in Materials Science and Engineering in 2005. He joined the Naval Research Lab in 2005 as a NRC postdoctoral fellow, where he focused on carbon nanotube synthesis and devices. During this time, the fever that is 2DMs was reborn, and Dr. Robinson began working on epitaxial graphene growth of silicon carbide. Subsequently, in 2007, Dr. Robinson moved back to Penn State to work for the Penn State Electro-Optics Center where he established projects in graphene and "beyond graphene" materials. The success of these programs enabled him to transition to the Penn State Materials Science and Engineering Department as an Assistant Professor and was promoted to an Associate Professor in 2015. He co-founded the Center for Two-Dimensional and Layered Materials and the NSF I/UCRC Center for Atomically Thin Multifunctional Coatings (ATOMIC) and currently serves as Co-Director of both. He is a 2015 NSF-CAREER recipient and has authored or coauthored over 200 peer reviewed journal publications with a significant focus on low dimensional electronic materials.

\section{Introduction}

In 2004, the state-of-the-art technique for realizing atomically thin materials consisted of a roll of tape and a layered crystal held together by van der Waals forces [1,2]. The so-called scotch-tape exfoliation method enabled isolation of molecularly thin layers from dozens of van der Waals [also known as twodimensional (2D)] materials such as molybdenum disulfide $\left(\mathrm{MoS}_{2}\right)$, tungsten diselenide ( $\left.\mathrm{WSe}_{2}\right)$, and hexagonal boron nitride $(\mathrm{hBN})[2,3]$. On realizing the promise of $2 \mathrm{D}$ materials (2DMs) for use in next-generation technologies, the community 
quickly advanced beyond micrometer-sized flakes of $2 \mathrm{D}$ layers produced by the scotch-tape method and toward the realization of continuous films over large-area substrates (up to $300 \mathrm{~mm}$ diameter $\mathrm{SiO}_{2} /$ silicon) $[4,5,6,7,8,9]$, all enabled by simple vapor deposition techniques [10]. The most rudimentary of these techniques utilizes a tube furnace-based approach, including solid source precursors and, more recently, nucleation or seeding agents that can yield 2D films over large areas $[4,11,12$, 13]. The beauty of this approach lies in its logistical simplicityone can merely heat source powders and substrates together in a tube furnace to achieve rapid synthesis of large-area 2D films in nearly any laboratory. This enables researchers to investigate fundamental material properties, basic physics, and proof-ofconcept devices with only a small barrier to creating the films themselves [10]. Following deposition, 2D films are commonly characterized via Raman, photoluminescence (PL), and X-ray photoelectron spectroscopies, which can be used to determine film composition, layer number, and band gap. Despite the ease of these deposition and characterization techniques, some limitations exist in their application to 2DMs [10]. Here, we highlight commonly overlooked scientific details of 2DMs synthesis and characterization that can ultimately impact scientific claims, with focus on solid-source and seed-promoted deposition, as well as Raman, PL, and X-ray photoelectron spectroscopic characterization.

\section{Chemical vapor deposition of 2-dimensional materials}

\section{Solid-source chemical vapor deposition}

The rapid development of the field of 2DMs synthesis research has been possible due to its relatively low cost of entry. For only a few thousand dollars, solid source precursors such as sulfur, tungsten-oxide, and molybdenum-oxide powders, and commonly available inventory, one can set up a simple tube furnace and begin growing 2DMs. While this tube furnace infrastructure dominates $2 \mathrm{DM}$ synthesis research today $[14,15,16]$, realization of truly repeatable, high-quality synthetic $2 \mathrm{DMs}$ requires significant modifications to solid-source (S) chemical vapor deposition (CVD) processes or completely different syntheses approaches. This is due to the poor reproducibility of S-CVD synthesis recipes, which often vary widely between furnaces and even between individual researchers in a single laboratory. Several variables of paramount importance can easily be overlooked during S-CVD processes, such as reactor and crucible geometry, furnace heating and cooling rates [17, 18], and substrate cleaning or surface preparation [5]. These variables are often unreported or overlooked in the literature, with many researchers claiming S-CVD to be a viable technique for uniform, large-scale 2DM synthesis. While some studies demonstrate quasi-uniform deposition on centimeter- scale samples, scaling to industrially relevant substrate sizes (>200 mm) using S-CVD is unlikely. Similarly, 2DM literature contains little mention of reactor "seasoning" or the effects of precursor buildup on crucibles and the interior of quartz tubes when S-CVD is utilized. This buildup significantly impacts material growth in hot wall S-CVD studies because it leads to a continuously changing partial pressure of precursors with each additional run, making every run unique [19]. Beyond incomplete reporting of reactor and process details in 2DM SCVD literature, S-CVD offers little control over precursor materials or concentration at the substrate surface. One commonly observed consequence of the horizontal tube furnace-based S-CVD approach is a deposition plume [Figs. 1(a) and $1(b)$ ], containing a gradient of 2D layer domain morphologies across the substrate surface. The quintessential triangular monolayer is often only observed only in a specific region of this deposition plume [Fig. 1(c)], due to variation in the precursor concentration across the substrate surface, and as a result, varying $2 \mathrm{D}$ crystal edge terminations and growth rates [20]. Control over precursor concentration in S-CVD studies is primarily achieved by varying source temperature and distance from substrate [20].

While S-CVD is a common technique for rapid realization of millimeter-scale 2DMs, the complexity of tube furnacebased S-CVD can lead to poor reproducibility even between personnel within a single research group. Therefore, process parameters reported for S-CVD techniques should be viewed merely as qualitative guidelines, where reported recipes are not likely to directly translate between laboratories but can serve as a valuable starting point for 2DM synthesis studies. One effort to reduce variability in S-CVD of 2DMs lies in computational investigations to understand flow patterns and precursor concentration variation at substrates surfaces $[17,18,21]$. While these efforts are relatively few, they can greatly aid understanding of the requirements for 2DM growth. Beyond SCVD growth of 2DMs, it is important to consider the use of alternative gas source deposition methods, such as atomic layer deposition [22], traditional CVD [4], and metal organic (MO) CVD [10], which offer controllable introduction of precursors into the reactors. Such techniques may require greater initial capital but can yield highly reproducible results and uniform, large-area 2D films. Efforts to achieve large-area films of 2DMs have matured in recent years, with demonstrations of coalesced films of transition metal dichalcogenides (TMDs) like tungsten diselenide $\left(\mathrm{WSe}_{2}\right)$ and $\mathrm{MoS}_{2}$ grown over centimeter scales $[4,5,6,7]$. These large-area films have been possible through the use of MOCVD, where the concentration and ratios of metal and chalcogen species can be precisely controlled via bubblers, and sequential nucleation and ripening steps may be performed to control TMD domain sizes and layer number $[5,6]$. 
(a)

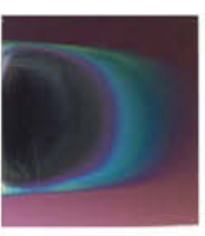

(b)

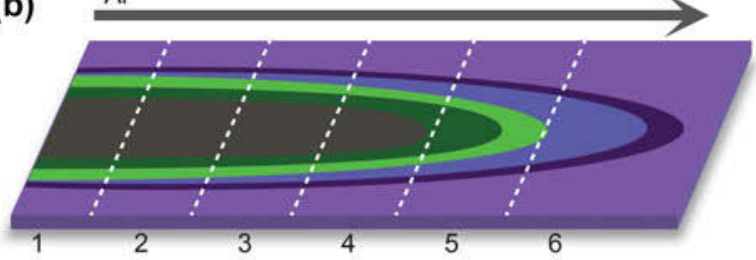

(c)
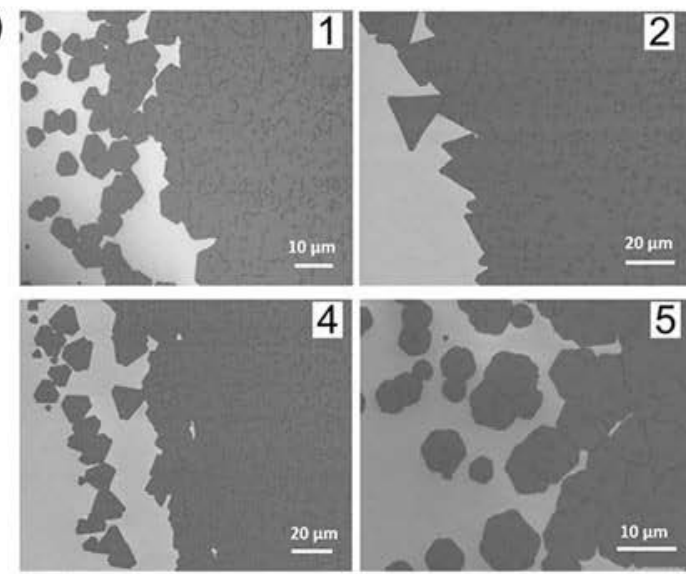

2
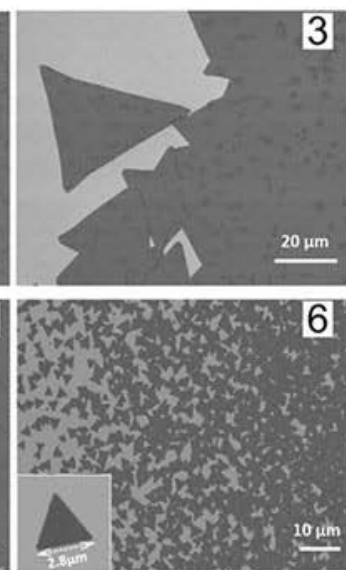

(d)

(e)

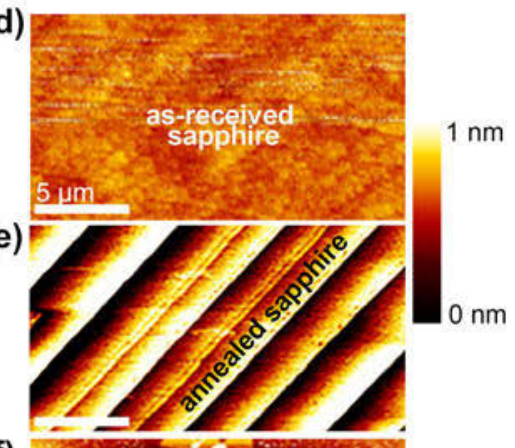

(f)

(g)

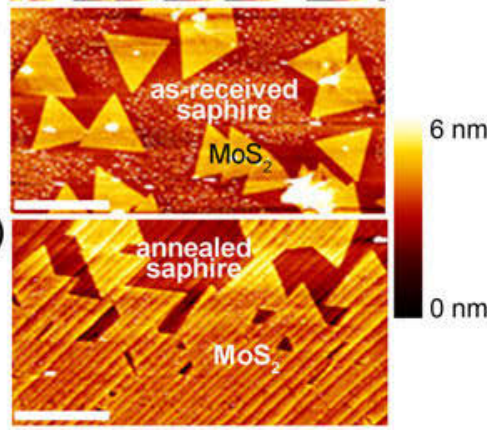

Figure 1: (a)-(c) $\mathrm{MoS}_{2}$ grown on $\mathrm{SiO}_{2} / \mathrm{Si}$ via S-CVD. (a) A typical $\mathrm{MoS}_{2}$ deposition plume, which can be divided into regions of varying MoS 2 morphologies (b). (c) Scanning electron microscope images of these regions. (d) The as-received c-plane sapphire. (e) c-plane sapphire annealed at $1150{ }^{\circ} \mathrm{C}$ for $8 \mathrm{~h}$ in ambient, resulting in the formation of terraces. (f) S-CVD-grown $\mathrm{MoS}_{2}$ on as-received sapphire showing no domain orientation. (g) S-CVD-grown MoS 2 on annealed sapphire showing orientation of domains. (a)-(c) reproduced with permission from Ref. 20.

Beyond the use of gas source deposition methods, substrate pretreatment can be used to control 2DM growth. This is shown in both MOCVD and S-CVD [Figs. 1(d)-1(g)] studies of TMD growth on c-plane sapphire [20]. While many 2DM synthesis studies utilize $\mathrm{SiO}_{2} / \mathrm{Si}$ substrates, c-plane sapphire is an increasingly popular substrate, due to potential for aligned 2DM domains resulting from long-range commensurability with sapphire $[5,6,7,23]$. Direct growth of $2 \mathrm{DMs}$ on asreceived c-plane sapphire, however, may not be sufficient in achieving oriented 2DM domains from S-CVD growth. Annealing sapphire to reduce the contact angle of the substrates is shown to enable dramatic improvement in domain orientation due to the formation of terraces in the sapphire surface, which promote certain domain orientations [Figs. 1(d)-1(g)] [5, 23, 24]. The use of c-plane sapphire as a substrate for epitaxial growth has enabled understanding of the requirements for lateral growth of domains and control of nominal layer number [6]. Observation of this domain orientation in both S-CVD and MOCVD studies reveals that substrate engineering can be an effective strategy to control the orientation and growth of $2 \mathrm{D}$ layers.

\section{Seed-promoted CVD}

In addition to the use of MOCVD for large-area 2DM growth, nucleation agents and seeding techniques have gained traction as tools to achieve increased $2 \mathrm{DM}$ domain sizes $[4,11,12,13$,
25]. Kang et al. [4] popularized the use of alkali-based compounds (e.g., $\mathrm{NaCl}$ ) to achieve large-domain 2D TMD layers via MOCVD, and the approach has since been expanded to a large library of 2D layers grown via S-CVD [26]. While salt-assisted growth does result in large-area TMD films with fewer grain boundaries, recent studies show $\mathrm{Na}$ atoms present at the TMD/substrate interface, which can lead to variation in coupling between the growth substrate and TMD layers. This causes spatial variation in electronic properties of the grown TMD [27]. Such variations in coupling can also affect the quality of the grown material significantly, resulting in a tensile strain in the layers and a reduction of the PL intensity of $\mathrm{MoS}_{2}$ layers by roughly $100 \times$. Furthermore, these studies indicate that while $\mathrm{NaCl}$ may serve to control nucleation and promote large area growth of $\mathrm{MoS}_{2}, \mathrm{NaCl}$ can also degrade the electrical performance of these grown semiconducting layers even when the films are transferred from the growth substrate [27]. More importantly, however, is the $50+$ years of research in the semiconductor industry that provides overwhelming evidence that the presence of alkali ions leads to higher oxide leakage currents and reliability problems in semiconductor devices when operated at high temperatures and voltages [28, 29, 30, 31, 32, 33].

This was demonstrated by Snow et al. [32] in 1965 (Fig. 2) through nonuniform shifts in capacitance-voltage $(\mathrm{C}-\mathrm{V})$ curves in a Na-contaminated metal-oxide-silicon (MOS) capacitor. The oxide leakage currents observed in the MOS structures are associated with the drift in $\mathrm{C}-\mathrm{V}$ curves. The ionic 

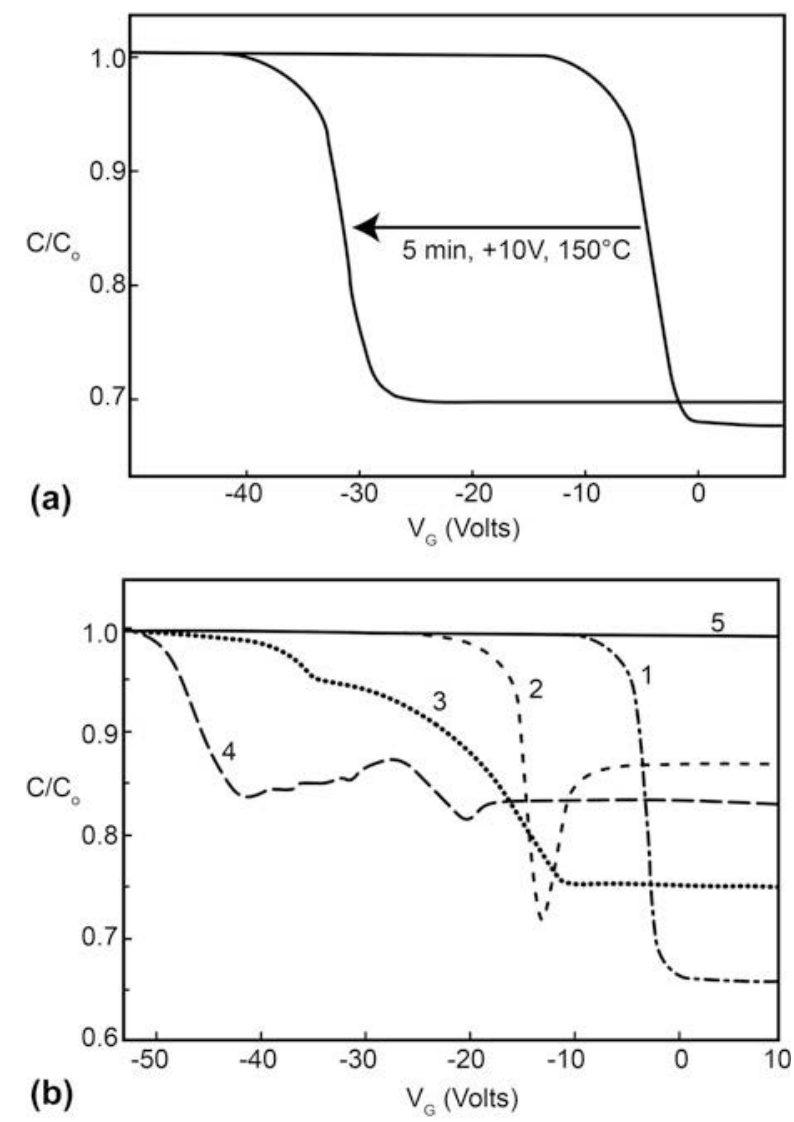

Figure 2: (a) C-V curves of an MOS structure with sodium contamination at the metal/oxide interface. The high diffusion rates of $\mathrm{Na}$ through the oxide under electrical bias lead to severe shifts in the CV curves, demonstrating significant device instability due to alkali ions. (b) C-V curves showing the more complicated curves due to nonuniform contamination over the surface of the device. Reproduced with permission from Ref. 32 .

drift within the oxide leads to significant shifts in the silicon charge, where upon application of a positive bias at $150{ }^{\circ} \mathrm{C}$ for 5 min, a $>100 \mathrm{~V}$ bias shift in the $\mathrm{C}-\mathrm{V}$ curve occurs [Fig. 2(a)], corresponding to a buildup of negative charge. This shift is independent of substrate doping. When $\mathrm{Na}$ and other alkali ions are not present in the MOS capacitors, the $\mathrm{C}-\mathrm{V}$ curves do not drift. As shown in Fig. 2(b), alkali-contaminated devices can also exhibit more complicated $\mathrm{C}-\mathrm{V}$ curves after drift. These distorted curves are caused by nonuniform alkali contamination over the surface of the device, causing some areas to drift more than others, leading to adjacent devices displaying different characteristics. Considering many integrated circuits operate between 55 and $85{ }^{\circ} \mathrm{C}$, one can expect significant diffusion of alkali ions and reduced transistor stability if such ions are present, even at low concentrations. Any commercial semiconductor fabrication facility will strongly object to a process that utilizes any alkali ion-based process, indicating that alkali ions must be avoided at all cost when considering 2DMs for augmenting semiconductor technology for complimentary metal-oxide-semiconductor (CMOS) applications. Other applications including low power devices for Internet of Things technology and 2DM-based chemical and biological sensors may have a higher tolerance for these mobile ions.

\section{Spectroscopic characterization of 2D materials}

\section{Raman and PL spectroscopies}

Detailed investigation of the quality of synthesized 2DMs requires a technique that examines the sample on an appropriate length scale and provides relevant structural or chemical information. Raman and PL spectroscopies are excellent characterization tools for $2 \mathrm{DMs}$ because they require little to no sample preparation and provide a wealth of information within short acquisition times [34, 35, 36, 37]. However, this extensive information can be easily misinterpreted if one is inexperienced in spectral analysis of inelastic scattering in 2DMs. One commonly misinterpreted result of spectral (Raman and PL) data relates to the identification of the number of layers in 2DMs. Generally, this misinterpretation is a result of limited spectral analysis and an unawareness that certain spectral features can be affected by factors unrelated to layer number (Fig. 3).

The most common method of reporting 2DM layer number is via Raman spectroscopy, based on the spectral separation of the $E^{1}{ }_{2 g}$ and $A_{1 g}$ vibrational modes in $\mathrm{MoS}_{2}$ [Fig. 3(a)], which increases with increasing layer number [38]. This approach is challenging due to the factors such as film strain [Fig. 3(b)] [39], concentration of sulfur vacancies [Fig. 3(c)] [40], and electronic carrier concentrations [Fig. 3(d)] [41]-all of which impact the absolute spacing between $\mathrm{E}_{2 \mathrm{~g}}$ and $\mathrm{A}_{1 \mathrm{~g}}$ in $\mathrm{MoS}_{2}$. Some of these characteristics like sulfur vacancy concentration can change with time or environment, whereas others like strain and carrier concentration are predominantly determined by the growth conditions. There are a few additional ways to utilize Raman for identifying 2D layer number, as noted in Fig. 3(e). Given access to a Raman spectrometer with ultra-low frequency Rayleigh filters and thus the ability to analyze $\mathrm{THz}$ frequencies (approximately spectral regions below $50 \mathrm{~cm}^{-1}$ ), one may use $\mathrm{THz}$ modes to confirm 2DM layer number. Raman peaks in this spectral range for van der Waals materials are associated with shear [C in Fig. 3(e)] and layer breathing [LB in Fig. 3(e)] modes and are therefore the most direct way of counting the number of 2D layers [42]. In addition, the $\mathrm{THz}$ bands are less susceptible to peak shift as a function of strain [43].

Another approach to layer number measurement-often more accessible than $\mathrm{THz}$ Raman-is the measurement of 2DM PL. Semiconducting monolayer TMDs typically exhibit an optical band gap in the visible spectrum. Measuring the PL of these materials requires the use of an excitation laser energy that is above the semiconductor optical band gap and 

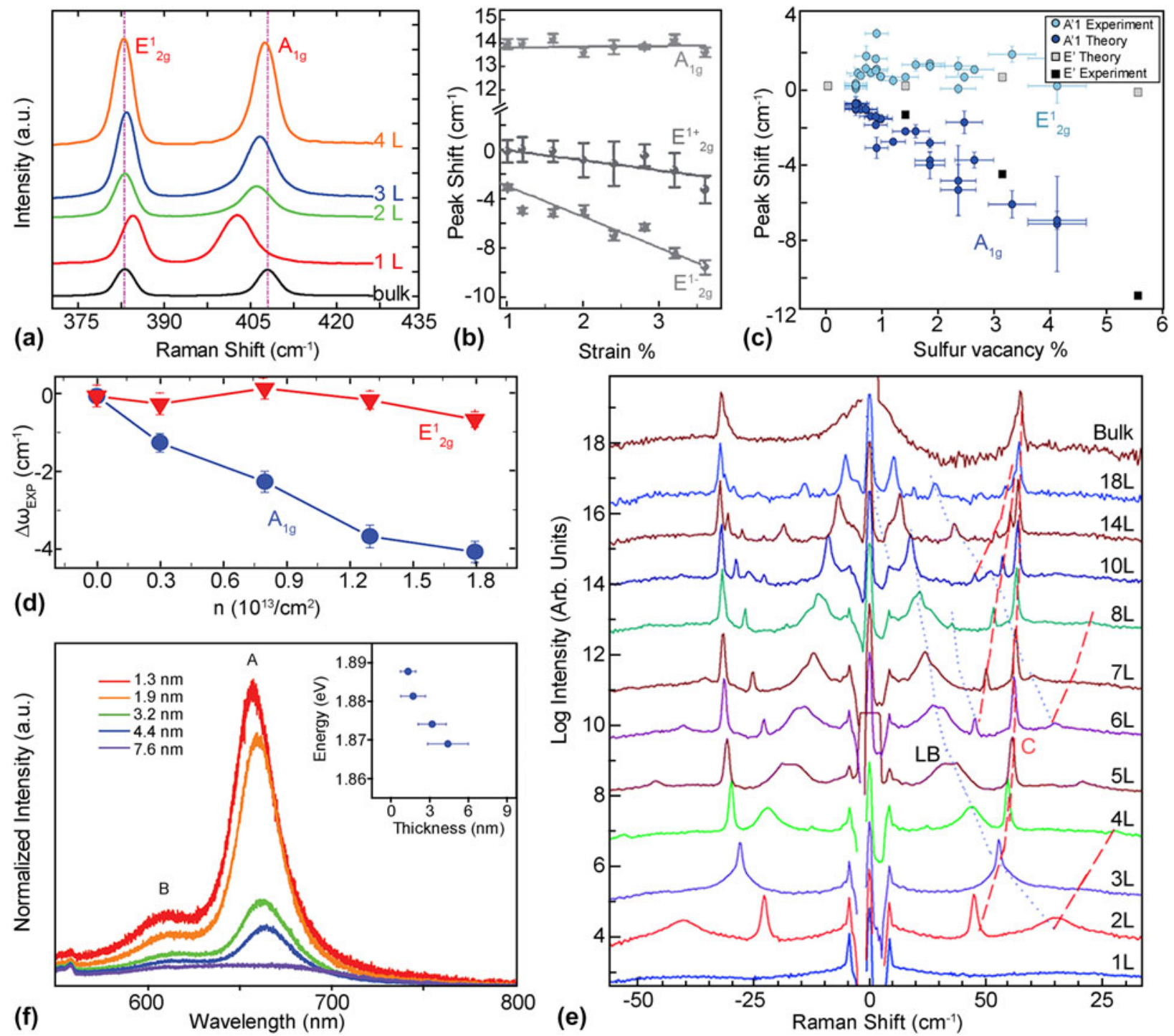

Figure 3: (a) $E_{2 g}$ and $A_{1 g}$ modes of $M_{0} S_{2}$, which shift with increasing $M_{0} S_{2}$ layer number. (b) Peak shift of $E_{2 g}^{1}$ and $A_{1 g}$ modes in strained MoS . The $E_{2 g}^{1}$ mode shifts to higher wavenumber when compressed and shifts to lower wavenumber under tension. (c) Theoretical calculations and experimental measurements of $M_{2} S_{2} E_{2 g}^{1}$ and $A_{1 g}$ peak shifts due to increasing sulfur vacancy \%. (d) Changes in $\operatorname{MoS}_{2} E_{2 g}^{1}$ and $A_{1 g}$ positions as a function of carrier concentration. (e) THz Raman measurements of $\mathrm{MoS}_{2}$, showing changes in the spectral region below $50 \mathrm{~cm}^{-1}$ as a function of $\mathrm{MoS}_{2}$ layer number. (f) MoS $\mathrm{PL}$ as a function of MoS $\mathrm{S}_{2}$ thickness (1.3-7.6 nm). (a)-(f) Reproduced with permission from Refs. 38, 39, 40, 41, 42, and 44.

a spectrometer that enables access to the PL energy [Fig. 3(f)]. Most research-grade Raman spectrometers match these conditions for $\mathrm{MoS}_{2}$, which has an indirect band gap of $\sim 1.3 \mathrm{eV}$ in bulk and a direct band gap of $\sim 1.8 \mathrm{eV}$ at monolayer thickness. Therefore, the PL peak position of $\mathrm{MoS}_{2}$ may be used as an additional indication of the layer number [44]. However, the PL response of the monolayer material is so sensitive that the peak rapidly and dynamically evolves in response to the environment-even when one merely breathes onto the sample (Fig. 4). Molecules adsorbed onto $\mathrm{MoS}_{2}$ layers from breathing onto the surface change the electronic band structure of the $\mathrm{MoS}_{2}$. One breath onto the sample changes the PL peak position by $20 \mathrm{meV}$. This peak position change is greater than that between $\mathrm{MoS}_{2}$ flakes with thicknesses of 1.3 and $7.6 \mathrm{~nm}$, as shown in Fig. 3(f) [44]. This "breath" experiment highlights the importance of understanding sample history and the environmental considerations when utilizing PL for quantification of layer thickness. Conversely, many groups have investigated the PL response of $2 \mathrm{DMs}$ as chemical sensors, leveraging this surface sensitivity while considering irradiation effects from the excitation laser $[45,46]$.

\section{X-ray photoelectron spectroscopy}

In addition to Raman and PL, X-ray photoelectron spectroscopy (XPS) can serve as a valuable 2DM characterization technique. XPS provides direct evidence of elemental bonding 

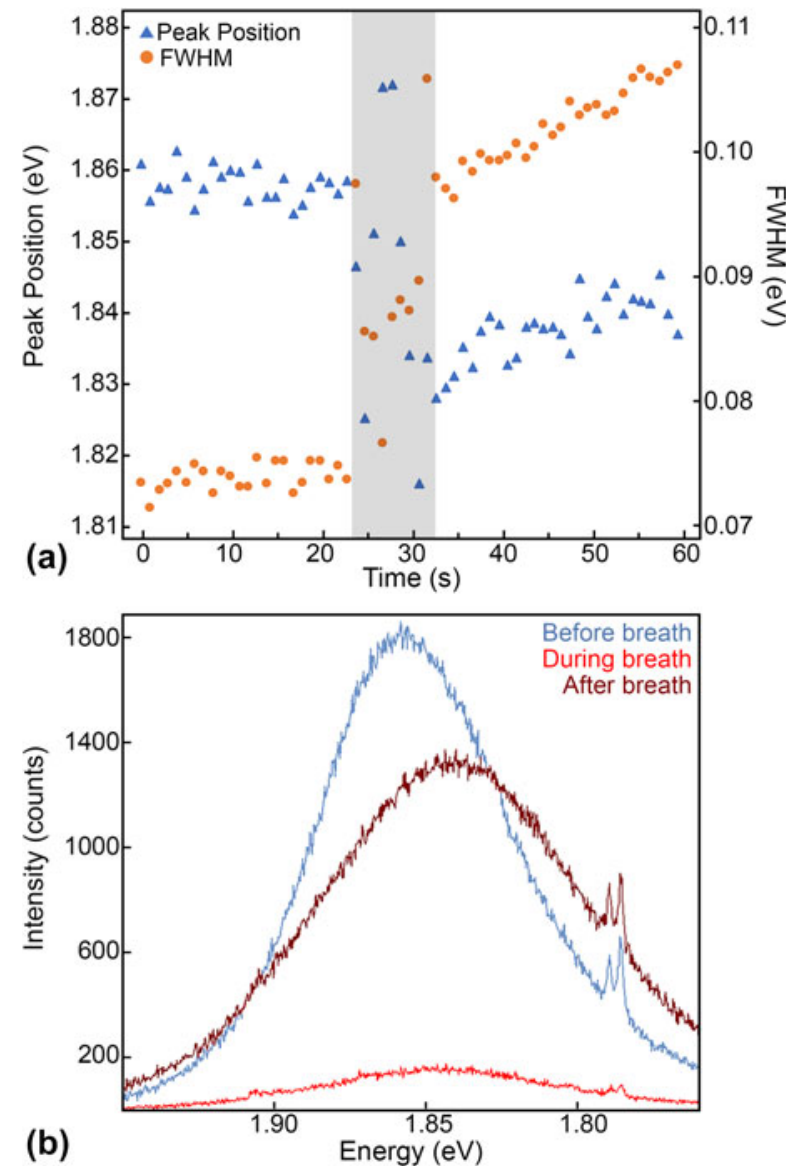

Figure 4: (a) PL response (highlighted in gray) of monolayer $\mathrm{MoS}_{2}$ as a result of a student breathing on the sample with "minty fresh breath." The PL peak position $(\triangle)$ decreases after the breath event (shaded region) and the FWHM $(O)$ increases. (b) Selected PL peaks at the time interval just before the breath event, during the breath, and $\sim 35 \mathrm{~s}$ after the breath. The doublet around 1.79 $\mathrm{eV}$ is a result of $\mathrm{Cr}^{+}$in the sapphire substrate.

at the surfaces of materials, making it attractive for understanding the composition of 2DMs. This technique has a unique combination of attributes that align particularly well with 2DMs, including surface sensitivity, accurate quantification with little or no matrix effects, sensitivity to every element in the periodic table except $\mathrm{H}$ and $\mathrm{He}$, and the ability to determine the nearest neighbor chemical environment in many instances. This technique is commonly used in the 2DM field to investigate chemistry and stoichiometry in $2 \mathrm{DMs}$, as well as dopant concentrations and impacts on valence band maxima (VBMs). To effectively utilize XPS for investigation of 2DMs, several considerations regarding stoichiometry and valence band measurements must be made.

Perhaps the attribute that XPS is best known for is its ability to determine the nearest neighbor chemical environment. The binding energy of core electrons emitted and detected via XPS is affected by formal valence state and other differences in chemical environment. Thus, XPS can be used to explore surface and interfacial reactions between 2DMs and to

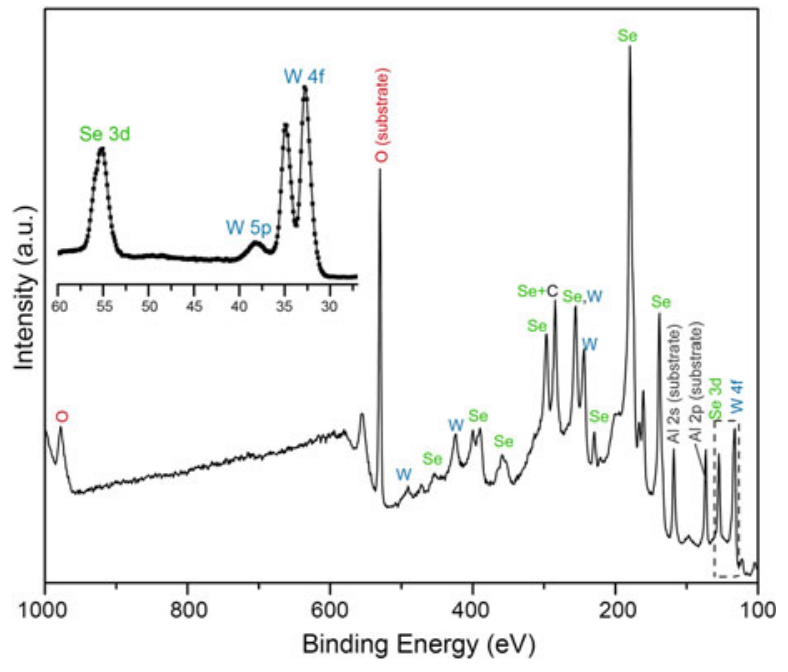

Figure 5: XPS spectra collected for WSe $\mathrm{W}_{2}$ grown on sapphire. W, Se, Al, and 0 peaks are indicated. High-resolution spectra (inset) show Se and W with a Se:W ratio of 1.96. This measured ratio may deviate significantly from the true Se:W ratio, as found from additional studies of exfoliated $\mathrm{WSe}_{2}$. These studies suggest that RSF derivation from references samples is required for accurate XPS analysis of 2DMs.

investigate relative elemental composition. Figure 5 shows a typical spectrum of $\mathrm{WSe}_{2}$ grown on sapphire $\left(\mathrm{Al}_{2} \mathrm{O}_{3}\right)$, where $\mathrm{W}, \mathrm{Se}, \mathrm{Al}$, and $\mathrm{O}$ peaks are clearly evident. The inset shows high-resolution spectra of the $\mathrm{W} 4 f$ and Se $3 d$ regions, and the measured Se:W ratio is found to be 1.96 (close to the expected 2.0). As a check of repeatability and accuracy of these measurements, a series of bulk 2DMs were repeatedly exfoliated and measured for composition (for flakes with acceptably low levels of $\mathrm{C}$ and $\mathrm{O}$ contamination). The standard deviation of the chalcogen-to-metal ratio varied by $<2 \%$ for the TMDs $\mathrm{MoS}_{2}$, $\mathrm{WS}_{2}$, WSe $e_{2}$, and the layered material $\mathrm{Bi}_{2} \mathrm{Te}_{3}$. The actual standard deviation of thin 2DMs on substrates is expected to be worse due to lower signal strength but will remain $<5 \%$.

The accuracy of these measured stoichiometries, however, is another matter entirely. In all cases, the bulk material composition and crystal chemistry were confirmed using Xray fluorescence and X-ray diffraction, respectively. The XPSmeasured stoichiometries of $\mathrm{MoS}_{2}$ and $\mathrm{WS}_{2}$ were within $3 \%$ of the true values. However, when this same methodology was applied to $\mathrm{WSe}_{2}$, the Se:W ratio was 1.69 instead of 2.00 , a $16 \%$ error. The Te:Bi ratio on $\mathrm{Bi}_{2} \mathrm{Te}_{3}$ was 1.19 instead of 1.50 . These results, obtained under ideal conditions, should provide a cautionary tale that determining the absolute stoichiometry of 2DMs may require derivation of relative sensitivity factors (RSFs) from reference samples rather than relying on RSFs supplied by the XPS instrument vendor. Under the best conditions of a continuous thin film of $\mathrm{MoS}_{2}$ on a substrate with no interfering peaks, the $2 \sigma$ standard deviation ( $95 \%$ confidence interval) of XPS-measured stoichiometry suggests that the best that can be achieved is $\mathrm{MoS}_{2.0 \pm 0.1}(5 \%)$. Therefore, 

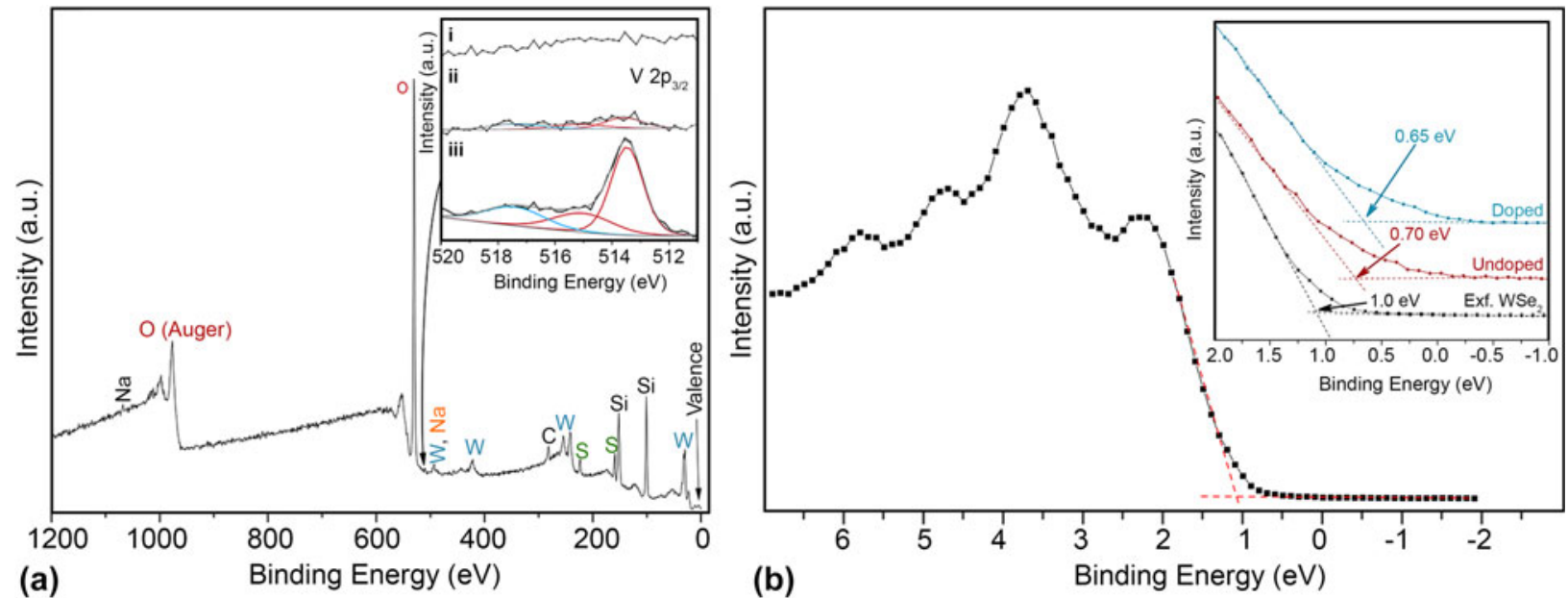

Figure 6: (a) XPS spectra of V-doped $\mathrm{WS}_{2}$ on sapphire. $\mathrm{SiO}_{2}$ signal dominates the spectra, accounting for $90 \%$ of the acquired signal. $<7 \%$ of the signal originates from $W, S$, and $V$. The inset in (a) shows the $V$ region for $(a, i)$ undoped, (a, ii) lightly doped $(W: V=41)$, and (a,iii) highly doped $(W: V=7.7)$ WS 2 . (a, iii) shows multiple chemical states of V. (b) VBM of exfoliated and Re-doped (inset) WSe $\mathrm{F}_{2}$. The measured valence band offset shown in the inset is found to be statistically insignificant with follow-up measurements. All spectra are charged referenced to $\mathrm{W}_{7 / 2}$ in $\mathrm{WSe}_{2}$ at $32.70 \mathrm{eV}$.

one should avoid claims of a stoichiometric 2DM based on XPS, as the error is likely $3-5 \%$ - a variation large enough to dramatically affect the optical and electronic properties.

Beyond measuring the stoichiometry of $2 \mathrm{DMs}$, there is intense interest in doping 2DMs to alter their electronic properties [10]. XPS is ideal for understanding the bonding environment of the dopants (e.g., dopant bonding to chalcogen or transition metal) but can also shed light on how the dopant impacts the electronic structure via measurement of VBMs. While the data used to identify bonding in XPS are based on core electrons, weakly bound valence electrons are also accessible. Measurements of the VBM can be combined with careful core level measurements to yield valence band offsets, which are important for understanding and predicting potential heterojunctions in $2 \mathrm{D}$ devices $[47,48]$. However, the X-ray cross section of these electrons is typically quite low, resulting in weak XPS signals [Fig. 6(a)]. This is evident from the spectra acquired from V-doped $\mathrm{WS}_{2}$ on $\mathrm{SiO}_{2}$ [Fig. 6(a)]. The sum of $\mathrm{W}, \mathrm{S}$, and $\mathrm{V}$ signals from this sample is $<7 \%$ of the total signal, and nearly $90 \%$ of the signal originates from the $\mathrm{SiO}_{2}$ substrate (with the balance coming from adsorbed organics). Furthermore, the V $2 p_{3 / 2}$ spectra of undoped, lightly doped, and highly doped $\mathrm{WS}_{2}$ [Fig. 6(a), inset] show evidence of multiple chemical states, requiring curve fitting into three overlapping components despite the fact that there is $<0.3 \%$ total vanadium present. The peak at $513.5 \mathrm{eV}$ is due to $\mathrm{VS}_{x}$ species with the higher binding energy peaks due to various oxides of vanadium. The lightly doped sample exhibits a $\mathrm{W}: \mathrm{V}$ of $\sim 41$, while the more heavily doped sample has a ratio of 7.7. In addition to these weak XPS signals, when films are placed on dielectric substrates, there is inevitable substrate charging that must be accounted for.
When determining VBMs in XPS, care must be taken to ensure that the acquired data are statistically significant. This is exemplified in Fig. 6(b), which shows measured VBMs for Redoped ( $\mathrm{W}: \mathrm{Re}=4$ ) and undoped $\mathrm{WSe}_{2}$ grown on sapphire. Freshly exfoliated bulk $\mathrm{WSe}_{2}$ is also shown for comparison. This provides a prime example of how Se vacancies can also lead to shifting in the VBM, even in undoped samples, where there is a $0.3 \mathrm{eV}$ shift between exfoliated $\mathrm{WSe}_{2}$ grown via chemical vapor transport and MOCVD grown $\mathrm{WSe}_{2}$ (undoped). The VBM is typically determined by finding the intersection of two linear fits of background near $0 \mathrm{eV}$ and the near-linear portion of the leading edge of the valence band [Fig. 6(b) inset]. Through this approach, a very small $(0.05 \mathrm{eV})$ difference is found between the doped and undoped $\mathrm{WSe}_{2}$ [Fig. 6(b)]. However, repeated measurements $(n=4)$ reveal that these values are not statistically different. By employing high-energy resolution scans with signal-to-noise ratio $>30: 1$, careful charge correction and careful fitting, a $2 \sigma$ standard deviation $\pm 0.10 \mathrm{eV}$, can be achieved. Thus, researchers must take care to acquire sufficiently precise and statistically significant measurements when investigating doping and VBM in 2DMs as the noise levels in these measurements can be much greater than the shifts themselves.

\section{Conclusion}

This article accumulates commonly overlooked scientific details in the synthesis and characterization 2DMs, with a focus on TMDs. It is intended to be used as a cautionary guide for researchers working in vapor-phase synthesis and spectroscopic characterization of 2DMs. Specifically, researchers should be aware of variability associated with S-CVD-grown 
materials and potential influences of nucleation agents on the properties of the synthesized 2DMs. When characterizing grown 2DMs, an understanding of the impacts of growth conditions and the local environment is crucial. Furthermore, reporting of 2DM layer number and stoichiometry must be done with caution if Raman and XPS techniques are used, and statistical significance of VBM measurements should be reported. As the $2 \mathrm{DM}$ field continues to progress, synthesis and characterization details must be scrutinized and communicated to researchers in the field at large to ensure consensus in scientific reporting and drawn conclusions.

\section{Acknowledgments}

S.S. and J.A.R. acknowledge the funding from NSF CAREER (Award: 1453924). N.B. and J.A.R. acknowledge the funding for this work that was provided by the Northrop Grumman Mission Systems' University Research Program, Semiconductor Research Corporation Intel/Global Research Collaboration Fellowship Program, task 2741.001, Pennsylvania State University Materials Research Institute's Material Characterization Laboratory. The authors would like to thank Dr. Kehao Zhang whose research results have been used in Figs. 1(d)-1(g). The authors would also like to acknowledge Azimkhan Kozhakmetov, Dr. Fu Zhang, and Prof. Mauricio Terrones who provided the samples for XPS measurements.

\section{References}

1. K.S. Novoselov, A.K. Geim, S.V. Morozov, D. Jiang, M.I. Katsnelson, I.V. Grigorieva, S.V. Dubonos, and

A.A. Firsov: Two-dimensional gas of massless Dirac fermions in graphene. Nature 438(7065), 197 (2005).

2. K.S. Novoselov, D. Jiang, F. Schedin, T.J. Booth, V. V Khotkevich, S. V Morozov, and A.K. Geim: Two-dimensional atomic crystals. Proc. Natl. Acad. Sci. U. S. A. 102(30), 10451 (2005).

3. A.K. Geim and I.V. Grigorieva: Van der Waals heterostructures. Nature 499(7459), 419 (2013).

4. K. Kang, S. Xie, L. Huang, Y. Han, P.Y. Huang, K.F. Mak, C.-J. Kim, D. Muller, and J. Park: High-mobility three-atom-thick semiconducting films with wafer-scale homogeneity. Nature 520(7549), 656 (2015).

5. Y.-C. Lin, B. Jariwala, B.M. Bersch, K. Xu, Y. Nie, B. Wang, S.M. Eichfeld, X. Zhang, T.H. Choudhury, Y. Pan, R. Addou, C.M. Smyth, J. Li, K. Zhang, M.A. Haque, S. Fölsch, R. M. Feenstra, R.M. Wallace, K. Cho, S.K. Fullerton-Shirey, J.M. Redwing, and J.A. Robinson: Realizing Large-Scale, Electronic-Grade Two-Dimensional Semiconductors. ACS Nano 12(2), 965 (2018).

6. X. Zhang, T.H. Choudhury, M. Chubarov, Y. Xiang, B. Jariwala, F. Zhang, N. Alem, G.-C. Wang, J.A. Robinson, and
J.M. Redwing: Diffusion-Controlled Epitaxy of Large Area Coalesced WSe2 Monolayers on Sapphire. Nano Lett. 18(2), 1049 (2018).

7. S.M. Eichfeld, L. Hossain, Y.-C. Lin, A.F. Piasecki, B. Kupp, A.G. Birdwell, R.A. Burke, N. Lu, X. Peng, J. Li, A. Azcatl, S. McDonnell, R.M. Wallace, M.J. Kim, T.S. Mayer,

J.M. Redwing, and J.A. Robinson: Highly Scalable, Atomically Thin WSe2 Grown via Metal -Organic Chemical Vapor Deposition. ACS Nano 9(2), 2080 (2015).

8. H. Jeong, D.Y. Kim, J. Kim, S. Moon, N. Han, S.H. Lee, O.F.N. Okello, K. Song, S.-Y. Choi, and J.K. Kim: Wafer-scale and selective-area growth of high-quality hexagonal boron nitride on $\mathrm{Ni}(111)$ by metal-organic chemical vapor deposition. Sci. Rep. 9(1), 5736 (2019).

9. J. Robertson, X. Liu, C. Yue, M. Escarra, and J. Wei: Wafer-scale synthesis of monolayer and few-layer MoS2 via thermal vapor sulfurization. 2D Mater. 4(4), 045007 (2017).

10. N. Briggs, S. Subramanian, Z. Lin, X. Li, X. Zhang, K. Zhang, K. Xiao, D. Geohegan, R. Wallace, L.-Q. Chen, M. Terrones, A. Ebrahimi, S. Das, J. Redwing, C. Hinkle, K. Momeni, A. van Duin, V. Crespi, S. Kar, and J.A. Robinson: A roadmap for electronic grade 2D materials. 2D Mater. 6(2), 022001 (2019).

11. H. Kim, D. Ovchinnikov, D. Deiana, D. Unuchek, and A. Kis: Suppressing Nucleation in Metal -Organic Chemical Vapor Deposition of MoS2 Monolayers by Alkali Metal Halides. Nano Lett. 17(8), 5056 (2017).

12. X. Ling, Y.-H. Lee, Y. Lin, W. Fang, L. Yu, M.S. Dresselhaus, and J. Kong: Role of the Seeding Promoter in MoS2 Growth by Chemical Vapor Deposition. Nano Lett. 14(2), 464 (2014).

13. S. Huang, L. Liang, X. Ling, A.A. Puretzky, D.B. Geohegan, B.G. Sumpter, J. Kong, V. Meunier, and M.S. Dresselhaus: LowFrequency Interlayer Raman Modes to Probe Interface of Twisted Bilayer MoS2. Nano Lett. 16(2), 1435 (2016).

14. Z. Cai, B. Liu, X. Zou, and H.-M. Cheng: Chemical Vapor Deposition Growth and Applications of Two-Dimensional Materials and Their Heterostructures. Chem. Rev. 118(13), 6091 (2018).

15. T. Zhang and L. Fu: Controllable Chemical Vapor Deposition Growth of Two-Dimensional Heterostructures. Chem 4(4), 671 (2018),

16. G.R. Bhimanapati, Z. Lin, V. Meunier, Y. Jung, J. Cha, S. Das, D. Xiao, Y. Son, M.S. Strano, V. R. Cooper, L. Liang, S.G. Louie, E. Ringe, W. Zhou, S.S. Kim, R.R. Naik, B.G. Sumpter, H. Terrones, F. Xia, Y. Wang, J. Zhu, D. Akinwande, N. Alem, J.A. Schuller, R.E. Schaak, M. Terrones, and J.A. Robinson: Recent Advances in Two-Dimensional Materials beyond Graphene. ACS Nano 9(12), 11509 (2015).

17. K. Momeni, Y. Ji, K. Zhang, J. A. Robinson, and L.-Q. Chen: Multiscale framework for simulation-guided growth of $2 \mathrm{D}$ materials. npj 2D Mater. Appl. 2(1), 27 (2018).

18. F. Zhang, K. Momeni, M.A. AlSaud, A. Azizi, M.F. Hainey, J.M. Redwing, L.-Q. Chen, and N. Alem: Controlled synthesis of 
2D transition metal dichalcogenides: from vertical to planar MoS2. 2D Mater. 4(2), 025029 (2017).

19. M.J. Hampden-Smith and T.T. Kodas: Chem. Vap. Depos. 1, 8 (1995).

20. S. Wang, Y. Rong, Y. Fan, M. Pacios, H. Bhaskaran, K. He, and J.H. Warner: Shape evolution of monolayer MoS2 crystals grown by chemical vapor deposition. Chem. Mater. 26(22), 6371 (2014).

21. Y. Xuan, A. Jain, S. Zafar, R. Lotfi, N. Nayir, Y. Wang,

T.H. Choudhury, S. Wright, J. Feraca, L. Rosenbaum,

J.M. Redwing, V. Crespi, and A.C.T. van Duin: Multi-scale modeling of gas-phase reactions in metal-organic chemical vapor deposition growth of WSe2. J. Cryst. Growth 527, 125247 (2019).

22. W. Hao, C. Marichy, and C. Journet: Atomic layer deposition of stable 2D materials. 2D Mater. 6(1), 012001 (2018).

23. K. Zhang, N.J. Borys, B.M. Bersch, G.R. Bhimanapati, K. Xu, B. Wang, K. Wang, M. Labella, T.A. Williams, M.A. Haque, E.S. Barnard, S. Fullerton-Shirey, P.J. Schuck, and

J.A. Robinson: Deconvoluting the Photonic and Electronic Response of 2D Materials: The Case of MoS2. Sci. Rep. 7(1) (2017).

24. D. Dumcenco, D. Ovchinnikov, K. Marinov, P. Lazić,

M. Gibertini, N. Marzari, O.L. Sanchez, Y.-C. Kung,

D. Krasnozhon, M.-W. Chen, S. Bertolazzi, P. Gillet,

A. Fontcuberta i Morral, A. Radenovic, and A. Kis: Large-Area Epitaxial Monolayer MoS2. ACS Nano 9(4), 4611 (2015).

25. K. Zhang, B. Jariwala, J. Li, N.C. Briggs, B. Wang, D. Ruzmetov, R.A. Burke, J.O. Lerach, T.G. Ivanov, M. Haque, R.M. Feenstra, and J.A. Robinson: Large scale 2D/3D hybrids based on gallium nitride and transition metal dichalcogenides. Nanoscale 10(1), 336 (2018).

26. J. Zhou, J. Lin, X. Huang, Y. Zhou, Y. Chen, J. Xia, H. Wang, Y. Xie, H. Yu, J. Lei, D. Wu, F. Liu, Q. Fu, Q. Zeng, C.-H. Hsu, C. Yang, L. Lu, T. Yu, Z. Shen, H. Lin, B.I. Yakobson, Q. Liu, K. Suenaga, G. Liu, and Z. Liu: A library of atomically thin metal chalcogenides. Nature 556(7701), 355 (2018).

27. K. Zhang, B.M. Bersch, F. Zhang, N.C. Briggs, S. Subramanian,

K. Xu, M. Chubarov, K. Wang, J.O. Lerach, J.M. Redwing,

S. K. Fullerton-Shirey, M. Terrones, and J.A. Robinson:

Considerations for Utilizing Sodium Chloride in Epitaxial Molybdenum Disulfide. ACS Appl. Mater. Interfaces 10(47), 40831 (2018).

28. P.L. Hefley and J.W. McPherson: in 26th Annu. Proc. Reliab. Phys. Symp. 1988 (IEEE, 1988), pp. $167-172$.

29. K. Schjølberg-Henriksen, G.U. Jensen, A. Hanneborg, and H. Jakobsen: Sodium contamination of $\mathrm{SiO} 2$ caused by anodic bonding. J. Micromechanics Microengineering 13(6), 845 (2003).

30. N. E. Lycoudes and C.C. Childers: Semiconductor Instability Failure Mechanisms Review. IEEE Trans. Reliab. R-29(3), 237 (1980).

31. M. Kuhn and D.J. Silversmith: Ionic Contamination and Transport of Mobile Ions in MOS Structures. J. Electrochem. Soc. 118(6), 966 (1971)
32. E. H. Snow, A.S. Grove, B.E. Deal, and C.T. Sah: Ion Transport Phenomena in Insulating Films. J. Appl. Phys. 36(5), 1664 (1965).

33. I. Constant, F. Tardif, and J. Derrien: Deposition and removal of sodium contamination on silicon wafers. Semicond. Sci. Technol. 15(1), 61 (2000).

34. J.-B. Wu, M.-L. Lin, X. Cong, H.-N. Liu, and P.-H. Tan: Raman spectroscopy of graphene-based materials and its applications in related devices. Chem. Soc. Rev. 47(5), 1822 (2018).

35. X. Zhang, Q.-H. Tan, J.-B. Wu, W. Shi, and P.-H. Tan: Review on the Raman spectroscopy of different types of layered materials. Nanoscale 8(12), 6435 (2016).

36. X. Zhang, X.-F. Qiao, W. Shi, J.-B. Wu, D.-S. Jiang, and P.-H. Tan: Phonon and Raman scattering of two-dimensional transition metal dichalcogenides from monolayer, multilayer to bulk material. Chem. Soc. Rev. 44(9), 2757 (2015).

37. J.-U. Lee and H. Cheong: Resonance Raman effects in transition metal dichalcogenides. J. Raman Spectrosc. 49(1), 66 (2018).

38. H. Li, Q. Zhang, C.C.R. Yap, B.K. Tay, T.H.T. Edwin, A. Olivier, and D. Baillargeat: From Bulk to Monolayer MoS2: Evolution of Raman Scattering. Adv. Funct. Mater. 22(7), 1385 (2012).

39. Y. Wang, C. Cong, C. Qiu, and T. Yu: Raman spectroscopy study of lattice vibration and crystallographic orientation of monolayer MoS2 under uniaxial strain. Small 9(17), 2857 (2013).

40. W.M. Parkin, A. Balan, L. Liang, P.M. Das, M. Lamparski, C.H. Naylor, J.A. Rodríguez-Manzo, A.T.C. Johnson, V. Meunier, and M. Drndić: Raman Shifts in Electron-Irradiated Monolayer MoS2. ACS Nano 10(4), 4134 (2016).

41. B. Chakraborty, A. Bera, D.V.S. Muthu, S. Bhowmick, U.V. Waghmare, and A.K. Sood: Symmetry-dependent phonon renormalization in monolayer MoS2 transistor. Phys. Rev. B 85(16), 161403 (2012).

42. J.-U. Lee, S. Woo, J. Park, H.C. Park, Y.-W. Son, and H. Cheong: Strain-shear coupling in bilayer MoS2. Nat. Commun. 8(1), 1370 (2017).

43. G. Eda, H. Yamaguchi, D. Voiry, T. Fujita, M. Chen, and M. Chhowalla: Photoluminescence from Chemically Exfoliated MoS2. Nano Lett. 11(12), 5111 (2011).

44. T. Pham, G. Li, E. Bekyarova, M.E. Itkis, and A. Mulchandani MoS2-Based Optoelectronic Gas Sensor with Sub-parts-per-billion Limit of NO2 Gas Detection. ACS Nano 13(3), 3196 (2019).

45. E. Akbari, K. Jahanbin, A. Afroozeh, P. Yupapin, and Z. Buntat: Phys. B Condens. Matter 545, 510 (2018).

46. S.A. Chambers, T. Droubay, T.C. Kaspar, and M. Gutowski Experimental determination of valence band maxima for $\mathrm{SrTiO} 3$, $\mathrm{TiO} 2$, and $\mathrm{SrO}$ and the associated valence band offsets with $\mathrm{Si}(001) . J$. Vac. Sci. Technol. B Microelectron. Nanom. Struct. 22(4), 2205 (2004).

47. M.-H. Chiu, C. Zhang, H.-W. Shiu, C.-P. Chuu, C.-H. Chen, C.-Y.S. Chang, C.-H. Chen, M.-Y. Chou, C.-K. Shih, and L.-J. Li: Determination of band alignment in the single-layer MoS2/WSe2 heterojunction. Nat. Commun. 6(1), 7666 (2015) 